\title{
Intersubband absorption in p-Ge/SiGe quantum wells grown on $\mathrm{Si}$
}

\author{
Kevin Gallacher ${ }^{\mathrm{a}}$, Andrea Ballabio ${ }^{\mathrm{b}}$, Ross W. Millar $^{\mathrm{a}}$, Antonio Samarelli $^{\mathrm{a}}$, Jacopo Frigerio $^{\mathrm{b}}$, Danny Chrastina $^{\mathrm{b}}$, \\ Giovanni Isella ${ }^{\mathrm{b}}$, Leonetta Baldassarre ${ }^{\mathrm{c}}$, Michele Ortolani ${ }^{\mathrm{c}}$, Emilie Sakat ${ }^{\mathrm{d}}$, Paolo Biagioni ${ }^{\mathrm{d}}$, Douglas J. Paul ${ }^{\mathrm{a}}$ \\ ${ }^{a}$ School of Engineering, University of Glasgow, U.K \\ ${ }^{\mathrm{b}}$ L-NESS, Politecnico di Milano, Como, Italy \\ ${ }^{c}$ Center for Life NanoScience@Sapienza, Istituto Italiano di Tecnologia, Rome, Italy \\ ${ }^{\mathrm{d}}$ Physics Department, Politecnico di Milano, Milano, Italy
}

Email: Douglas.Paul@glasgow.ac.uk

\begin{abstract}
Intersubband absorption from p-Ge quantum wells grown on $\mathrm{Si}$ is demonstrated. The absorption can be tuned by adjusting the quantum well thickness. FTIR transmission measurements on as-grown wafers show broad absorption corresponding to intersubband transitions.
\end{abstract}

\section{INTRODUCTION}

There is an increased interest to develop Si based detectors that cover the important transmission windows within the midinfrared (3-5 and $8-12 \mu \mathrm{m}$ wavelength) to enable on-chip biological and gas sensing spectroscopy. Currently the gold standard for detection at these wavelengths is $\mathrm{HgCdTe}$ due to its inherently large detectivities from interband absorption [1]. Disadvantages with $\mathrm{HgCdTe}$ is that it is fragile, has low process uniformity, and is not easily integrated with $\mathrm{Si}$. Therefore, to realise on-chip sensors an alternative is required. A good candidate is quantum well (QW) infrared photodetectors. Since absorption can occur from intersubband transitions within the QW, the absorption can be tuned by adjusting the well width, thereby changing the energy of the confined subband states. This has been previously demonstrated with $\mathrm{SiGe}[2,3]$. Due to the advent of new growth technologies such as low energy plasma enhanced chemical vapour deposition (LEPECVD), it is now possible to grow high quality Ge QWs on a SiGe strain relaxed virtual substrate [4]. This paper demonstrates, to-date, the first experimentally observed intersubband absorption for $\mathrm{p}-\mathrm{Ge}$ QWs grown on $\mathrm{Si}$.

\section{DESIGN AND MODELLING}

The design consists of p-type compressively strained Ge QWs of either 4, 5, or $6 \mathrm{~nm}$ thickness, strain symmetrised with $\mathrm{Si}_{0.5} \mathrm{Ge}_{0.5}$ barriers. This allows the growth of a potentially unlimited number of periods without ever exceeding critical thickness limitations, thereby increasing overall absorption. Another benefit of p-Ge QWs is that it should provide larger absorption coefficients compared to p-SiGe designs, due to the smaller effective masses of the holes. In addition, by using the more complex non-parabolicty and strong coupling of the valence bands, this relaxes selection rules and allows both TE (x-y) and TM (z) polarization. Hence, both surface normal and waveguide geometry devices can be realised. The band energies and confined wavefunctions were calculated using a self-consistent 6-band k.p Poisson-Schrodinger solver with the deformation potentials from reference [5]. Figure 1 shows the calculated band structure for a $5 \mathrm{~nm}$ Ge QW and $3.1 \mathrm{~nm}$ $\mathrm{Si}_{0.5} \mathrm{Ge}_{0.5}$ barriers. It is clear that the ground state in the $\mathrm{QW}$ is $\mathrm{HH} 1$ due to strain splitting of the $\mathrm{HH}$ and $\mathrm{LH}$ bands. The $\mathrm{HH} 2$, LH1, and HH3, LH2 are at roughly the same energies within the QW.

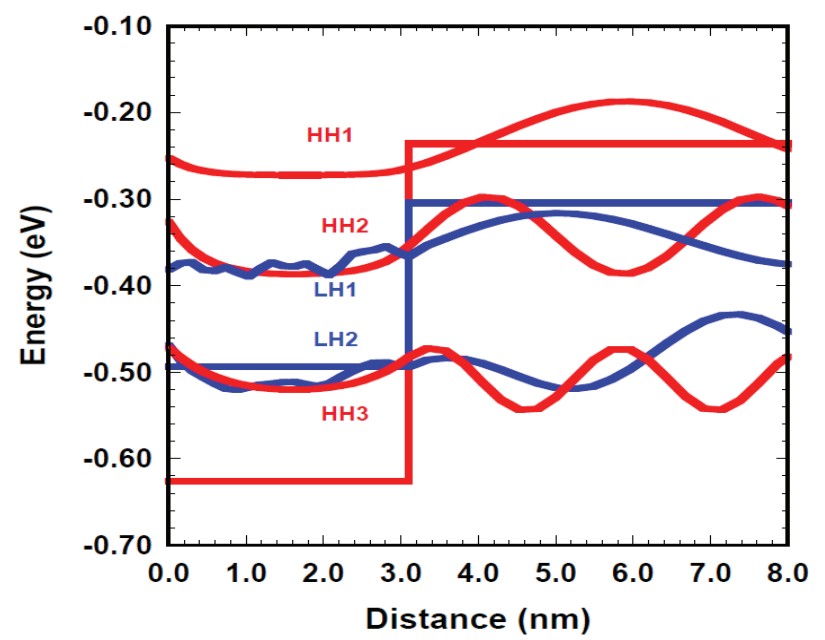

Figure 1. The calculated band structure for a $5 \mathrm{~nm}$ wide Ge quantum well, showing the squared wave functions for the lowest energy subband states for the heavy hole $(\mathrm{HH})$ and light hole $(\mathrm{LH})$ bands.

\section{GROWTH}

The designed Ge QW structures were grown on a Si substrate by LEPECVD. As previously stated, this growth technique allows the realisation of $\mathrm{Ge}$ rich superlattice structures. A $600 \mathrm{~nm}$ linearly graded buffer from $\mathrm{Si}$ to $\mathrm{Si}_{0.2} \mathrm{Ge}_{0.8}$ was first grown, followed by a $400 \mathrm{~nm} \mathrm{p}-\mathrm{Si}_{0.2} \mathrm{Ge}_{0.8}$ bottom contact region $\left(\mathrm{N}_{\mathrm{A}}=5 \times 10^{18} \mathrm{~cm}^{-3}\right)$. Then an undoped $10 \mathrm{~nm} \mathrm{Si}_{0.2} \mathrm{Ge}_{0.8}$ spacer region was grown followed by the active region consisting of over 400 periods of either 4 . 5 , or $6 \mathrm{~nm}$ doped QWs $\left(\mathrm{N}_{\mathrm{A}}=\right.$ 
$5 \times 10^{18} \mathrm{~cm}^{-3}$ ) sandwiched between $3.1 \mathrm{~nm} \mathrm{Si}_{0.5} \mathrm{Ge}_{0.5}$ barriers. Lastly, another undoped $10 \mathrm{~nm} \mathrm{Si}_{0.2} \mathrm{Ge}_{0.8}$ spacer layer was grown, followed by a $20 \mathrm{~nm} \mathrm{p}-\mathrm{Si}_{0.2} \mathrm{Ge}_{0.8}$ top contact region. Graded buffer layers and the fully strained state of the superlattice were observed by x-ray diffraction (XRD). Figure 2 shows the XRD spectra for the $6 \mathrm{~nm}$ QW structure. The $\mathrm{Si}(004)$ peak of the substrate is visible at $\mathrm{q}=7.365 \mathrm{~nm}^{-1}$ and is used as a reference. Superlattice fringes are visible with a spacing that indicates a period of $\sim 10 \mathrm{~nm}$. The zeroth-order fringe at $7.051 \mathrm{~nm}^{-1}$ is matched to the virtual substrate and indicates an average Ge content of $80 \%$ in the active layer stack.

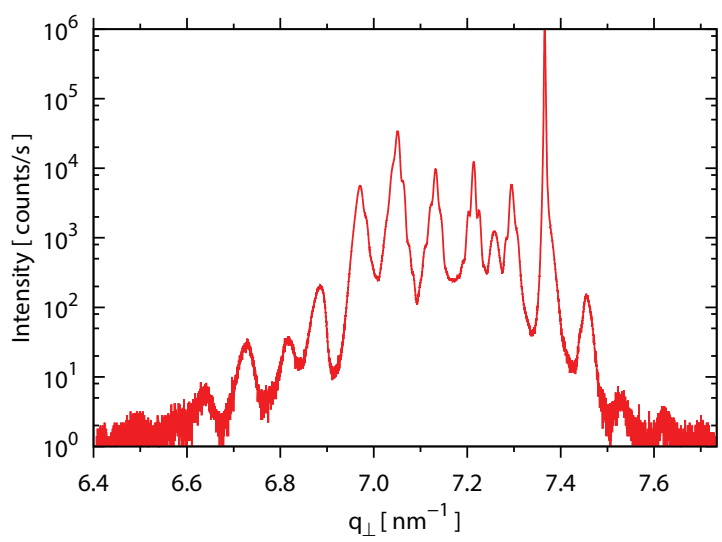

Figure 2. X-ray diffraction curves for the $6 \mathrm{~nm}$ wide Ge quantum well structure with $3.1 \mathrm{~nm}$ SiGe barriers grown on a Si substrate.

\section{CHARACTERISATION}

Fourier transform infrared (FTIR) transmission measurements were performed on the as-grown wafers in vacuum at temperatures down to $6 \mathrm{~K}$. The setup consisted of a Bruker IFS $66 \mathrm{v} / \mathrm{s}$ spectrometer with a $\mathrm{SiC}$ glow bar as the MIR source and a $\mathrm{LN}_{2}$ cooled $\mathrm{HgCdTe}$ detector. Blank chips of the grown wafers were diced and then bonded onto a cold finger of an Oxford Instruments closed cycle optical cryostat for surface normal transmission. Figure 3 (a) shows the transmission spectra at $300 \mathrm{~K}$ for the as-grown structures with QW widths of 4,5 , and $6 \mathrm{~nm}$. The overall low transmission of each spectra is due to the strong free carrier absorption from the heavily doped structures. The absorption peak at $\sim 9 \mu \mathrm{m}$ wavelength, which is visible in all the spectra, corresponds to oxygen impurities within the Si substrate. Each QW structure exhibits broad absorption. There is a clear absorption shift to lower wavelengths for thinner QWs, which is expected, due to the higher energy transitions between confined subband states. From the modelling, this corresponds to transitions between HH2-HH3, HH2-LH2, and LH1-LH2. This illustrates how the absorption can be tuned by changing the QW widths. Figure 3 (b) shows the low temperature transmission spectra of the $6 \mathrm{~nm}$ QW structure. It is clear that as the temperature decreases the absorption is increasing. There is negligible blue shift observed, due to the small temperature dependence on intersubband transitions compared to interband.
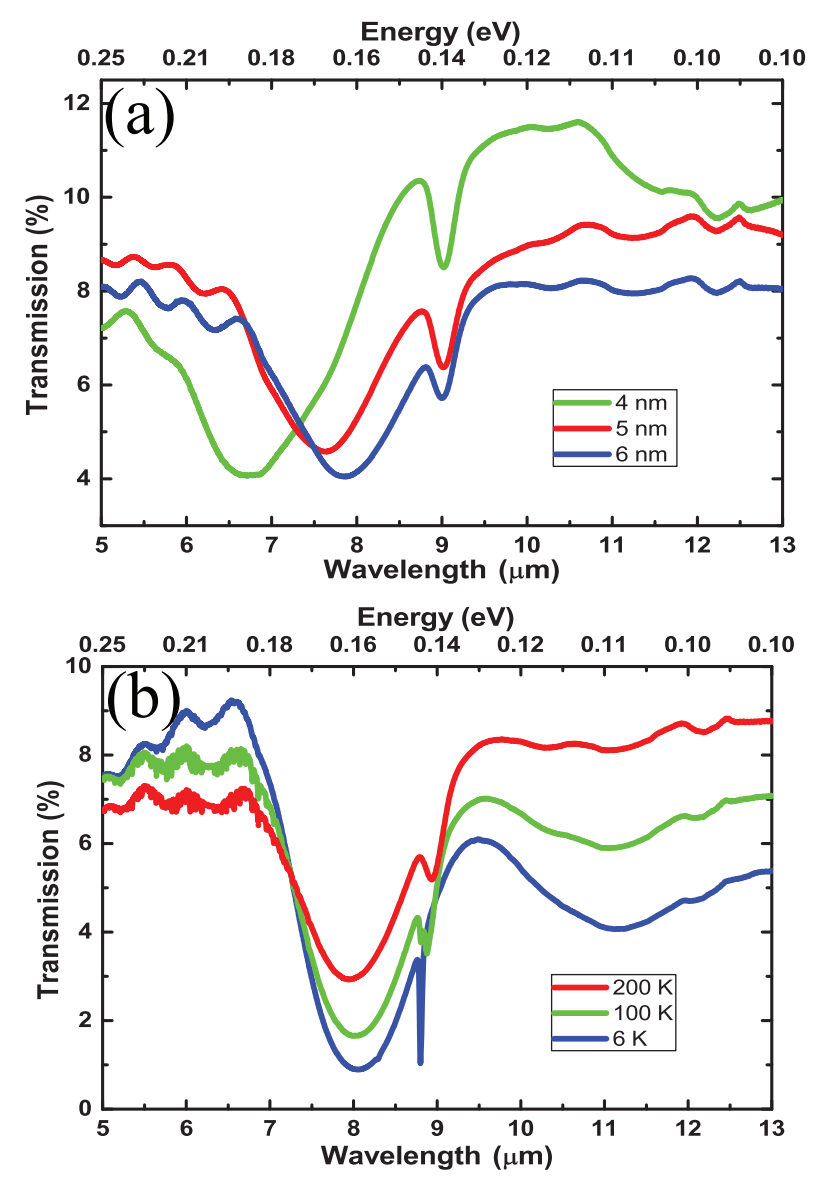

Figure 3. (a) FTIR transmission spectra at $300 \mathrm{~K}$ under vacuum for the as-grown 4, 5, and $6 \mathrm{~nm}$ wide Ge QW structures. (b) Low temperature FTIR transmission spectra of the $6 \mathrm{~nm}$ QW structure.

\section{CONCLUSION}

Intersubband absorption of $\mathrm{p}-\mathrm{Ge} / \mathrm{SiGe} \mathrm{QWs}$ with different widths has been demonstrated. Band structure modelling has been undertaken and designs have been grown. High quality growth of the Ge QWs has been confirmed through XRD analysis. FTIR transmission measurements in TE (surface normal) polarization show broad absorption that shifts to lower wavelengths for thinner QWs. It is envisaged that such designs could produce waveguide coupled photodetectors for on-chip spectroscopic sensing in the MIR.

\section{REFERENCES}

[1] Martyniuk, P., et al., New concepts in infrared photodetector designs. Applied Physics Reviews, 2014. 1(4)

[2] Chun, S.K., D.S. Pan, and K.L. Wang, INTERSUBBAND TRANSITIONS IN A P-TYPE DELTA-DOPED SIGE/SI QUANTUM-WELL. Physical Review B, 1993. 47(23): p. 15638-15647.

[3] De Seta, M., et al., Conduction band intersubband transitions in Ge/SiGe quantum wells. Applied Physics Letters, 2009. 95(5).

[4] Chrastina, D., et al., Thin relaxed SiGe virtual substrates grown by lowenergy plasma-enhanced chemical vapor deposition. Journal of Crystal Growth, 2005. 281(2-4): p. 281-289

[5] Paul, D.J., 8-band k.p modeling of the quantum confined Stark effect in Ge quantum wells on Si substrates. Physical Review B, 2008. 77 